\title{
The irreducible photon
}

\author{
David L. Andrews \\ School of Chemical Sciences, University of East Anglia, \\ Norwich NR4 7TJ, United Kingdom
}

\begin{abstract}
In recent years it has become evident that the primary concept of the photon has multiple interpretations, with widely differing secondary connotations. Despite the all-pervasive nature of this concept in science, some of the ancillary properties with which the photon is attributed in certain areas of application sit uneasily alongside those invoked in other areas. Certainly the range of applications extends far beyond what was envisaged in the original conception, now entering subjects extending from elementary particle physics and cosmology through to spectroscopy, statistical mechanics and photochemistry. Addressing this diverse context invites the question: What is there, that it is possible to assert as incontrovertibly true about the photon? Which properties are non-controversial, if others are the subject of debate? This paper describes an attempt to answer these questions, establishing as far as possible an irreducible core of what can rightly be asserted about the photon, and setting aside some of what often is, but should never be so asserted. Some of the more bewildering difficulties and differences of interpretation owe their origin to careless descriptions, highlighting a need to guard semantic precision; although simplifications are frequently and naturally expedient for didactic purposes, they carry the risk of becoming indelible. Focusing on such issues, the aim is to identify how much or how little about the photon can be regarded as truly non-controversial.
\end{abstract}

Keywords: Photonics, quantum electrodynamics, photon momentum, photon spin, photon angular momentum, photon wavefunction, quantum fields, multipolar radiation

\section{INTRODUCTION}

In recent years it has become evident that the fundamental concept of the photon has a multitude of physical interpretations, many with widely differing secondary connotations. Despite the all-pervasive and foundational nature of this key concept in science, some of the ancillary properties with which the photon is attributed in certain areas of application sit somewhat uneasily alongside those that are invoked in other areas. This partly reflects the fact that the range of applications has expanded strikingly far beyond what was envisaged in the original conception, now entering into subjects extending from elementary particle physics and cosmology through to spectroscopy, statistical mechanics and photochemistry.

In many respects the concept of the photon has become one whose detailed interpretation is strongly dependent on context. This conference series has repeatedly underscored the fact that the totality is now something much broader and deeper, and in some respects less readily definable, than has been admitted in the past.[1] It is too easy to become oblivious to a certain mystery that still surrounds the nature of this quantum of light, yet at heart the underlying theory has to relate unambiguously to scientific fact.

This paper represents an attempt to establish, at least as a basis for discussion, an irreducible core of what rightly can be asserted about the photon. To this end, key elements of a quantum electrodynamical theory are deployed to characterize an internally consistent set of physical properties that are also amenable to measurement. The study also aims to expose the weaknesses of certain common, but fundamentally untenable assertions concerning photon properties. Particular attention is paid to the attribution of wavefunctions and multipolar character to individual photons, each of which is logically unsustainable. While some associated statements and developments have obvious didactic purpose, it is all too easy for simplifications to become ingrained in a kind of unspoken folklore of the subject, imperceptibly moving beyond

*david.andrews@physics.org

7421-7 V. 1 (p.1 of 9) / Color: No / Format: A4 / Date: 7/8/2009 7:21:43 AM

SPIE USE: DB Check, Prod Check, Notes: 
the sphere of normal critical appraisal. It will be evident that some of the more bewildering difficulties and differences of photon interpretation owe their origin to careless narratives. In the textual depictions of any scientific concept there is arguably as much need to seek semantic precision, as there is to deploy theoretical representations that are mathematically correct.

\section{PHOTON ATTRIBUTES: THEORETICAL FOUNDATIONS}

The quantum theory of light, as widely understood, provides a formal and mathematically well-behaved construct for the representation of all photonic measurables, including 'ancillary' attributes of the photon such as its linear momentum and spin, electric and magnetic fields. However, even for these, there is not a complete consensus on certain aspects of their physicality. Before proceeding, it is therefore prudent to secure a suitably robust, rigorous and non-contentious basis for assessing the validity of these and various other photon attributes.

Quantum electrodynamics (QED) is a stringently tested quantum theory of light that satisfies all the necessary criteria.[2] QED treats matter and light on a common basis. It is universally agreed that the detailed behaviour of matter can only be explained using a quantum basis; it is a premise which remains 'unfalsified' in the Popperian sense, and it therefore is only logical to apply the same principles to light. As such, QED is internally consistent, treating light and matter as one whole system within which energy can be exchanged. It is, of course, also a theory that generates results with unequalled precision, as nicely exhibited in recent work by Gabrielse et al. on the magnetic moment of the electron and the fine structure constant, achieving $0.70 \mathrm{ppb}$ precision.[3] Moreover, a strong case can be made that QED is the only theory in which the concept of the photon has genuine legitimacy.

It is not proposed to rehearse the detailed formulation of QED at this juncture, but the pieces of theory that follow are firmly based in that formalism, informed by its conceptual perspective.

\subsection{A quantum of electromagnetic radiation}

To address the detailed issues, it is reasonable and appropriate to begin with the defining premise that each photon is a quantum of electromagnetic radiation. It is generally understood that the core meaning of the latter statement relates specifically to a quantization of energy - although energy is not the only physical quantity to be thus quantized. The Planck relation, $\Delta E=h v$, first established a simple relationship between the frequency $(v=c / \lambda)$ of optical radiation and a difference in energy between discrete states involved in an atomic transition. Einstein's subsequent treatment of the photoelectric effect, explaining the frequency threshold for photoelectric emission, incorporated the same quantum energy in the relation $T=h v-\phi$ for the kinetic energy of each photoelectron ( $\phi$ being the surface work function). This success, in particular, effected a paradigm shift in the interpretation of the energy quantum, furnishing it with particlelike attributes - notwithstanding the wave nature apparent from the stipulation of an optical frequency.

Although it may no longer seem entirely noteworthy, a hidden corollary to each of these interpretations was an assumption that what we now term 'photons' have a directly multiplicative energy - so that $n$ photons relate to an energy $n h v$. It is entirely for this reason, for example, that the Planck relationship later proved readily adaptable to laser multiphoton absorption, for which $\Delta E=n h v$. At a fundamental level, however, what this multiplicative quality signifies is that, for radiation of a given optical frequency, the quantized energy levels for electromagnetic radiation are equally spaced - which is of course by no means to be taken for granted in a quantum system (recall by contrast the spacing of levels in the hydrogen atom). It can, indeed, be shown that this feature arises directly as a result of the electromagnetic energy being quadratically dependent on its electric and magnetic fields. To conceive of the light energy quanta as having any kind of particle-like attribute is only possible as a result of the equal spacing of energy levels.

\subsection{Enumerator of a radiation mode}

On recognition that electromagnetic radiation is comprehensible in terms of photons, it is evident that these quanta must individually carry the signature properties of the light itself. Photons may be regarded as principally characterized by frequency, but they must also be distinguishable by a direction of propagation $\hat{\boldsymbol{k}}$ and polarization. For freely radiating 
light the three Cartesian components of $\boldsymbol{k}$ and the polarization variable constitute four degrees of freedom. ${ }^{\dagger}$ The combination of these features serves to establish a mode of the radiation field, usually defined by a wave-vector $\boldsymbol{k}=(2 \pi / \lambda) \hat{\boldsymbol{k}}$ (determining both the direction of propagation and the optical frequency) and a polarization parameter $\eta$. Embracing this interpretation, the number of photons $n$ that have this character within a given volume of space $V$ is thus interpreted as the occupancy of the radiation mode $(\boldsymbol{k}, \eta)$. The corresponding state of the radiation field has a quantum wavefunction which can be represented in Dirac notation by the ket $|n(\boldsymbol{k}, \eta)\rangle$.

For each radiation mode, the quantum energy levels form a set with a constant spacing $h v$, which in the context of quantum optics is more commonly written as $\hbar \omega$ (circular frequency $\omega=2 \pi v$ ). Connecting adjacent levels it is possible to define two operators; one is a photon annihilation operator, $a^{(\eta)}(\boldsymbol{k})$, operating to deliver a state with one less photon; the other is a photon creation operator, $a^{\dagger(\eta)}(\boldsymbol{k})$, delivering a state with one more photon. These designations colour, with particle connotations, the more widely used 'lowering' and 'raising' descriptors of quantum operator algebra. These operators act as follows on the radiation states;

$$
\begin{gathered}
a^{(\eta)}(\boldsymbol{k})|n(\boldsymbol{k}, \eta)\rangle=n^{1 / 2}|(n-1)(\boldsymbol{k}, \eta)\rangle, \\
a^{\dagger(\eta)}(\boldsymbol{k})|n(\boldsymbol{k}, \eta)\rangle=(n+1)^{1 / 2}|(n+1)(\boldsymbol{k}, \eta)\rangle .
\end{gathered}
$$

Moreover the two operators have a commutation relation given by:

$$
\left[a_{\boldsymbol{k}}^{(\eta)}, a_{\boldsymbol{k}^{\prime}}^{\dagger \eta^{\prime}}\right]=\delta_{\boldsymbol{k}, \boldsymbol{k}^{\prime}} \delta_{\eta, \eta^{\prime}}
$$

Before proceeding further it is worth remarking that, in the sense of consistency with the core principles of quantum electrodynamics, even the existence of a photon wavefunction proves problematic. A robust case for this assertion is given in section 2.8 .

\subsection{Conveyer of electric and magnetic fields}

As befits their dynamical status in a fully quantized description of electromagnetic radiation, the associated variables the transverse electric and magnetic fields - are promoted to operator status, being directly expressible in terms of the photon creation and annihilation operators. Explicitly, each is cast in terms of a summation over radiation modes:[4]

$$
\begin{aligned}
& \boldsymbol{e}^{\perp}(\boldsymbol{r})=i \sum_{\boldsymbol{k}, \eta}\left(\frac{\hbar c k}{2 \varepsilon_{0} V}\right)^{1 / 2}\left\{\boldsymbol{e}^{(\eta)}(\boldsymbol{k}) a^{(\eta)}(\boldsymbol{k}) e^{i \boldsymbol{k} \boldsymbol{r} r}-\overline{\boldsymbol{e}}^{(\eta)}(\boldsymbol{k}) a^{\dagger(\eta)}(\boldsymbol{k}) e^{-i \boldsymbol{k} \boldsymbol{r} \boldsymbol{r}}\right\}, \\
& \boldsymbol{b}(\boldsymbol{r})=i \sum_{\boldsymbol{k}, \eta}\left(\frac{\hbar c k \mu_{0}}{2 V}\right)^{1 / 2} \hat{\boldsymbol{k}} \times\left\{\boldsymbol{e}^{(\eta)}(\boldsymbol{k}) a^{(\eta)}(\boldsymbol{k}) e^{i \boldsymbol{k} . \boldsymbol{r}}-\overline{\boldsymbol{e}}^{(\eta)}(\boldsymbol{k}) a^{\dagger(\eta)}(\boldsymbol{k}) e^{-i \boldsymbol{k} . \boldsymbol{r}}\right\} .
\end{aligned}
$$

For simplicity, the above field expansions are given in a vacuum formulation. .Here $\boldsymbol{r}$ is a position vector, $\boldsymbol{e}^{(\eta)}(\boldsymbol{k})$ is the unit polarization vector for the radiation mode $(\boldsymbol{k}, \eta) ; \varepsilon_{0}$ is the vacuum permittivity and $\mu_{0}$ the vacuum permeability.

${ }^{\dagger}$ In some optically engineered beams of light the four modal degrees of freedom may be served by a different set; for example in Laguerre-Gaussian beams (which impose a twisted wave-front with respect to a defined direction of propagation) the set comprises the polarization, the wave-vector magnitude and two integers that designate the field distribution by a specific Laguerre polynomial. 
Also in (5) an overbar denotes complex conjugation - the polarization vector on which it appears is generally considered a complex quantity to entertain circular polarizations. The involvement of the ladder operators in (4) and (5) signifies that each electric or magnetic action of light either creates or destroys a photon. For example the electric field is engaged quadratically in conventional light scattering (where one photon annihilated and another created in a different mode).

It is useful to note at this stage that both fields (4) and (5) are readily derivable from a similarly cast mode expansion of the transverse electromagnetic vector potential;

$$
\boldsymbol{a}^{\perp}(\boldsymbol{r})=\sum_{\boldsymbol{k}, \eta}\left(\frac{\hbar}{2 \varepsilon_{0} c k V}\right)^{1 / 2}\left\{\boldsymbol{e}^{(\eta)}(\boldsymbol{k}) a^{(\eta)}(\boldsymbol{k}) e^{i \boldsymbol{k} \boldsymbol{r}}+\overline{\boldsymbol{e}}^{(\eta)}(\boldsymbol{k}) a^{\dot{\dagger}(\eta)}(\boldsymbol{k}) e^{-i \boldsymbol{k} \boldsymbol{r}}\right\},
$$

consistent with the fundamental relations $\boldsymbol{e}(\boldsymbol{r})=-\partial_{t} \boldsymbol{a}(\boldsymbol{r})$ and $\boldsymbol{b}(\boldsymbol{r})=\nabla \times \boldsymbol{a}(\boldsymbol{r})$, the time-derivative being effected in the time-explicit retarded form in which $\boldsymbol{k} . \boldsymbol{r} \rightarrow \boldsymbol{k} . \boldsymbol{r}-\omega t$.

\subsection{The zero-photon state has a finite energy for each radiation mode}

The energy density of electromagnetic radiation has the expression;

$$
\mathcal{H}_{\mathrm{rad}}(\boldsymbol{r})=\frac{1}{2}\left\{\varepsilon_{0} \boldsymbol{e}^{\perp 2}(\boldsymbol{r})+\mu_{0}^{-1} \boldsymbol{b}^{2}(\boldsymbol{r})\right\}
$$

manifestly comprising electric and magnetic contributions. Expressed in operator form using the field expansions (4) and (5), the radiation Hamiltonian is also concisely expressible as the following mode sum:

$$
\mathcal{H}_{\mathrm{rad}}(\boldsymbol{r})=\sum_{\boldsymbol{k}, \eta}\left(a^{\dagger(\eta)}(\boldsymbol{k}) a^{(\eta)}(\boldsymbol{k})+\frac{1}{2}\right) \hbar \omega / V
$$

Reverting to the form exhibited in (7), each contribution - electric and magnetic - can be evaluated individually, for each mode of the radiation field. For example, the electric contribution delivers;

$$
\begin{aligned}
\langle\mathcal{H}\rangle_{\mathrm{elec}}^{n(\boldsymbol{k}, \eta)} & =\frac{1}{2}\left\langle n(\boldsymbol{k}, \eta)\left|\varepsilon_{0} e^{\perp 2}(\boldsymbol{r})\right| n(\boldsymbol{k}, \eta)\right\rangle \\
& =\frac{\hbar c k}{4 V}\left\langle n(\boldsymbol{k}, \eta)\left|\left\{a^{(\eta)}(\boldsymbol{k}) a^{\dagger(\eta)}(\boldsymbol{k})+a^{\dagger(\eta)}(\boldsymbol{k}) a^{(\eta)}(\boldsymbol{k})\right\}\right| n(\boldsymbol{k}, \eta)\right\rangle .
\end{aligned}
$$

Terms quadratic in the creation or annihilation operators vanish through the orthogonality of states with different photon occupancy. The result is independent of position (within the quantization volume $V$ ). Moreover, from the result of applying equations (1) and (2) we have $a^{\dagger(\eta)}(\boldsymbol{k}) a^{(\eta)}(\boldsymbol{k})|n(\boldsymbol{k}, \eta)\rangle=\hat{n}^{(\eta)}(\boldsymbol{k})|n(\boldsymbol{k}, \eta)\rangle=n$, and using the commutation relation (3), we therefore obtain;

$$
\langle\mathcal{H}\rangle_{\text {elec }}^{n(\boldsymbol{k}, \eta)}=\frac{(2 n+1) \hbar c k}{4 V}=\frac{1}{2}\left(n+\frac{1}{2}\right) \hbar c k / V
$$

Precisely the same result ensues for the magnetic field contribution to (7); in a vacuum the energy is equally shared between electric and magnetic contributions. Then, since $c k=\omega$, we have;

$$
\langle\mathcal{H}\rangle^{n(\boldsymbol{k}, \eta)}=\left(n+\frac{1}{2}\right) \hbar \omega / V .
$$


Equation (11) exhibits the fact that within a volume $V$, each radiation mode has a finite ground-state zero-photon (vacuum) energy of $1 / 2 \hbar \omega$, usually interpreted in terms of vacuum fluctuations. In free space, of course, the lack of boundary conditions allows the set of radiation modes - and hence the associated vacuum energy - to be infinite. The above derivation shows that the non-vanishing ground state energy is a direct physical consequence of the lack of commutativity between the photon creation and annihilation operators, the latter fact a property reflecting the bosonic character of the photon. In addition to this background energy, (11) further signifies that $n$ photons of circular frequency $\omega$ are associated with an energy density $\hbar \omega / V$, consistent with the basic premise concerning photon energy.

\subsection{A quantum of linear momentum}

Thus far the focus has largely been on energy, to which the foundational Planck relation specifically refers. However there are grounds for considering this to reflect a more extensive principle, the quantization of electromagnetic radiation itself. Indeed, the principle of quantization does apply to other attributes of the radiation - notably the linear momentum and angular momentum. The first is readily addressed through the Poynting vector, classically defined by $\boldsymbol{S}(\boldsymbol{r})=\mu_{0}^{-1}\{\boldsymbol{e}(\boldsymbol{r}) \times \boldsymbol{b}(\boldsymbol{r})\}$. The optical linear momentum per unit volume, $\mathcal{P}(\boldsymbol{r})$ is expressible in terms of the Poynting vector through $\mathcal{P}(\boldsymbol{r})=\boldsymbol{S}(\boldsymbol{r}) / c^{2}$. Cast in its quantum field operator representation, assuming no static fields are present, the corresponding expectation value for the radiation state $|n(\boldsymbol{k}, \eta)\rangle$ is readily evaluated from (4) and (5), exploiting the relation $\varepsilon_{0} \mu_{0}=c^{-2}$; the result again proves to be independent of position within the quantization volume.

$$
\langle\mathcal{P}\rangle^{\eta(\boldsymbol{k}, \eta)}=\varepsilon_{0}\left\langle n(\boldsymbol{k}, \eta)\left|\boldsymbol{e}^{\perp}(\boldsymbol{r}) \times \boldsymbol{b}(\boldsymbol{r})\right| n(\boldsymbol{k}, \eta)\right\rangle=n(\boldsymbol{k}, \eta) \hbar \boldsymbol{k} / V
$$

Strictly, since the transverse electric and magnetic fields do not commute, the operator in (12) should be symmetrised as $\frac{1}{2}\left[\left\{\boldsymbol{e}^{\perp}(\boldsymbol{r}) \times \boldsymbol{b}(\boldsymbol{r})\right\}-\left\{\boldsymbol{b}(\boldsymbol{r}) \times \boldsymbol{e}^{\perp}(\boldsymbol{r})\right\}\right]-$ see ref. [5]. The given result arises just the same.

Equation (9) establishes that within a void of volume $V, n$ photons of wave-vector $\boldsymbol{k}$ deliver a linear momentum density of $n \hbar \boldsymbol{k} / V$, signifying that each photon has a linear momentum $\hbar \boldsymbol{k}$. It is less straightforward to assert the corresponding result for the linear momentum of a photon travelling in a dispersive medium, where there has been a long-standing controversy over the applicability of different formulations generally attributed to Abraham and Minkowski. The former delivers a photon momentum inversely scaled by the refractive index at the appropriate optical frequency; the latter formula scales the photon momentum in linear proportion to the refractive index. Resolution of the conundrum depends on giving a careful identification of meaning to each quantity, differentiating the different parts of a material system on which measurements of linear momentum of associated radiation forces are performed.[6-10]

\subsection{A quantum of angular momentum}

Just as mechanical angular momentum for a particle of unit mass at position $\boldsymbol{q}$ with respect to an origin is delivered by the vector cross product $\boldsymbol{q} \times \partial_{t} \boldsymbol{q}$, the spin angular momentum of light is delivered, as a volume density, by the product $\varepsilon_{0}\left\{\boldsymbol{a}^{\perp}(\boldsymbol{r}) \times \boldsymbol{e}^{\perp}(\boldsymbol{r})\right\}$. Evaluating the contribution for a specific radiation mode, in the quantized field representation we have;

$$
\begin{gathered}
\langle\mathcal{L}\rangle^{n(\boldsymbol{k}, \eta)}=\varepsilon_{0}\left\langle n(\boldsymbol{k}, \eta)\left|\boldsymbol{a}^{\perp}(\boldsymbol{r}) \times \boldsymbol{e}^{\perp}(\boldsymbol{r})\right| n(\boldsymbol{k}, \eta)\right\rangle=\left\{n(\boldsymbol{k}, \eta)+\frac{1}{2}\right\} \boldsymbol{\sigma}^{(\eta)}(\boldsymbol{k}) \hbar / V \\
\boldsymbol{\sigma}^{(\eta)}(\boldsymbol{k})=i\left\{e^{(\eta)}(\boldsymbol{k}) \times \bar{e}^{(\eta)}(\boldsymbol{k})\right\} .
\end{gathered}
$$

The eigenstates of the spin operator are radiation states having circular polarization, for which the results for each helicity are;

$$
\boldsymbol{e}^{(L / R)}(\boldsymbol{k})=\frac{1}{\sqrt{2}}(\hat{\boldsymbol{i}} \pm \hat{\boldsymbol{j}}) ; \quad \boldsymbol{\sigma}^{(L / R)}(\boldsymbol{k})= \pm \hat{\boldsymbol{k}}
$$


where $\hat{\boldsymbol{i}}$ and $\hat{\boldsymbol{j}}$ denote unit vectors for a pair of axes $(x, y)$ orthogonal to the propagation direction, in the usual righthanded sense. Thus, from (13), for a beam of a given wave-vector comprising $n_{L}$ and $n_{R}$ photons of left- and righthanded polarization, respectively, the net spin angular momentum is given by $\hbar \hat{\boldsymbol{k}}\left(n_{L}-n_{R}\right)$; see ref.[11], p. 490. The result is consistent with each left-circular photon conveying a spin angular momentum of +1 , and -1 for each righthanded one.

The conclusion over spin angular momentum is commonly cited as a manifestation of the boson character of the photon. Nonetheless, no such interpretation of the spin angular momentum is possible for other, perfectly admissible radiation states having different forms of polarization. For example a single-photon plane polarized state represents a quantum superposition of left- and right-handed states. Tackling spin angular momentum thus represents a point of departure from the irreducible core of properties that can be addressed with rigorous, incontrovertible assertions.

In fact, the above expression (13) for spin angular momentum is only one component of a complete angular momentum whose operator is constructed as a cross-product of the position and linear momentum, the latter delivered by the Poynting vector - see ref.[12], page 8;

$$
\hat{\mathcal{L}}=\varepsilon_{0} \int d^{3} r\left[\boldsymbol{r} \times\left\{\boldsymbol{e}^{\perp}(\boldsymbol{r}) \times \boldsymbol{b}(\boldsymbol{r})\right\}\right]
$$

comprising not just photon spin but also what has become known as an orbital angular momentum. The orbital term is a property of the radiation mode structure rather than individual photons.[13,14] Accordingly, it transpires that not just integer but even fractional values of the orbital angular momentum per photon are attainable.[15,16] As is the case with linear momentum, care also has to be taken to correctly address the case of photon angular momentum for light propagating into a dielectric medium. $[9,17]$

\subsection{The photon is massless}

The statement that a photon has no mass appears incontrovertible. A photon having a precisely zero mass is, inter alia, the only interpretation consistent with basic relativistic dynamics, as in the equation $E^{2}=m_{0}^{2} c^{4}+p^{2} c^{2}$. Occasional mentions in the literature of photons that are referred to as massive, as a result of dispersive engagement in light-matter interactions - see for example ref.[18] p.29, seemingly reflect non-standard and unnecessary interpretations. Other claims that the vacuum photon 'actually' has a mass, but one that is too small to be experimentally registered, belong in the category of untestable theories, failing Popper's criteria.

\subsection{A photon has no wavefunction}

The ostensibly surprising assertion that there is no such thing as a photon wavefunction can be thrown into better light on contrasting this statement with the truism that the quantum state of a radiation field with one photon does indeed have a wavefunction - as does a state with any number of photons. The difference between the two statements, seemingly minor when cast in words, is nonetheless important - and easy to see once it is pointed out. Consider any specific radiation mode $(\boldsymbol{k}, \eta)$. There is in fact no way of representing the wavefunction for the two-photon state, $|2(\boldsymbol{k}, \eta)\rangle$, as any kind of product of one-photon $|1(\boldsymbol{k}, \eta)\rangle$ state wavefunctions. This is not surprising; in the same way, it is not possible to represent the wavefunction for a $2 s$ electron as any product of $1 s$ wavefunctions. In the radiation case, to do so would also misrepresent the vacuum energy, which does not scale with $n$ but is exactly $1 / 2 \hbar \omega$ for both the $|1(\boldsymbol{k}, \eta)\rangle$ and $2(\boldsymbol{k}, \eta)\rangle$ states. This feature seems to undermine attempts to portray the quintessentially quantum mechanical nature of the photon by reference to 'its' wavefunction. $[19,20]$

There is a concise measurement-based interpretation, ascribed to one of the pioneers of optical coherence theory, that runs as follows: 'A photon is what a photodetector does'. In the spirit of this succinct depiction certain calculational methods have been demonstrated that can deliver a spatially localized visualization of photon propagation - see for example ref.[21] Such representations may indeed have some didactic value. Nonetheless, a more accurate description of what any conventional (or quantum) photodetector does, is that it responds to the vector potential field of impinging radiation, registering its quantum energy. Although it is in principle possible to simultaneously measure, for example, the energy, linear momentum and spin angular momentum of a photon - in the sense that the corresponding quantum 
field operators commute amongst themselves - in practice it is only one attribute of the quantized radiation that is thus registered. Measurement that is localized by the physical extent of a conventional detector must and should not be interpreted as signifying a localization of the photon itself; the very process of registration generally involves photon capture, thereby effecting a collapse of the state function for the radiation field. Specifically designed 'non-demolition' measurements on the optical field are arguably a different matter, but it is seldom claimed that these genuinely interrogate individual photons.[22,23] It is salutary to recall the view of Mandel and Wolf who, in their definitive work, [11] point out that attempts for whatever purposes to build a picture of 'localized photons' are fraught with danger, for 'a photon has no precise position no matter what the state may be'.

\section{DISCUSSION}

The contextual heritage of the photon context is strikingly captured by the commutation relationship (3), between the creation and annihilation operators for optical radiation modes. This is a relationship that underpins almost all of the expressions for derivative, measurable properties - moreover its form is the hallmark of bosonic character, pointing to an integer spin angular momentum. This establishes a connection with the status of the photon as an elementary particle; it is commonly listed as such in most particle physics textbooks - see for example p.3 in ref.[24]. The 'particle' designation does, of course, obscure the equally important wave nature of light, alluding in a potentially misleading way to material physicality.

Here, and in its original conception, it is notable that the photon concept first arose in contexts where the mode occupation number $n$ is seldom much different from 0 or 1 . Indeed this remains true for the vast majority of applications, in subjects extending from elementary particle physics to photochemistry. Although it is primarily in laser connections that higher levels of mode occupancy arise, it is remarkable that most of the key features concerning optical coherence and quantum optics can be addressed without deployment of the term 'photon'. In ref. [11], for example, the concept is only introduced half-way through the extensive treatise. In assessing the value of the photon concept in this particular discipline, it is also worth recalling that the phase properties of optical radiation stand in an uncertainty relationship with mode occupancy - so that states with a well-defined number of photons are necessarily low in recoverable phase information.[25] Again, the photon concept is invaluable in connection with non-classical radiation states, yet it sits uneasily alongside the recent discovery of polarization states that evolve during the free propagation of light.[26]

It is tempting to advise interpretive caution when the term 'photon' is used with an adjectival qualification. Previously, for example, it has been noted that the term 'virtual photon', designating a photon that is not itself measurable, is seamlessly extendable to all photons.[27]. Indeed, the authors or ref;[24] (p.140) observe that: 'In a sense every photon is virtual, being emitted and then sooner or later absorbed'. Now, the validity of the designation of photons by multipolar order, as for example in the term 'quadupolar photon' - see for example p.16 in ref.[28] - is being questioned.[29] The issues here are quite distinct from any that might surround usage of the term 'quadrupole radiation"; the latter is undoubtedly legitimate since it designates an angular distribution in emission that reflects the nature of the decay transition, at least in most atomic systems. However at the quantum level such spatial distributions can only be regarded as statistical, serving to identify the relative probabilities of emitted photons propagating in different directions.

Although the spectroscopic rules governing multipole transitions and angular momentum are non-controversial, an unguarded application of these same rules to the history of any individual photon, in a connected sequence of excited state decay and photodetection, can create apparent paradoxes. Consider for example an atom which, initially in an excited state, radiatively decays through an electric-dipole forbidden, electric-quadrupole allowed transition. In certain sections of the literature it appears to be assumed, if not always explicitly stated, that electric quadrupole decay transitions produce photons of a distinct character - yet it emerges that there is no sound theoretical basis for such a supposition. Although radiant emission of a given multipolar origin is in principle identifiable from the characteristic form of its spatial distribution, it does not follow that multipolar origination of any specific form should translate into a distinction in nature of the individually emitted photons. Specifically, if radiation emitted in an electric quadrupolar transition is detected by a conventional (electric dipolar) photodetector, then time-reversal arguments would indicate a

$¥$ In English the noun 'radiation' is ambiguous - it can mean either the act of radiating, or it can refer to that which is radiated. The same ambiguity is present in the Russian original.[30] 
different, logically incompatible character for the photon involved in the time-reversed, yet fully-allowed process. Nonetheless, the photon histories involved in quadrupole-emission, dipole-detection and its converse both have wellcharacterized quantum electrodynamical descriptions.[31,32] It has to be concluded that there is no clear physical meaning to the term 'quadrupole photon'.

The principle that electric quadrupolar emission is detectable by a regular dipolar detector is not only of fundamental importance, it is in principle testable. Many well-studied atomic spectral lines are uniquely attributable to electric quadrupole transitions, such as the ${ }^{1} S_{0} \leftarrow{ }^{1} D_{2}$ transition in $\mathrm{O}^{2+}$. The challenge is simple; ignoring relative intensities, could the radiation emitted in the course of such a decay be experimentally differentiated from the dipolar emission of a different source, at the same wavelength, using the same detection setup? An answer in the negative appears to be unequivocal.

In conclusion, based on a quantum electrodynamical foundation, this paper has attempted to identify several core attributes of photons which are mutually compatible and physically incontrovertible. Even amongst those which are most familiar, such as linear and angular momentum, there are issues that remain to be resolved. Caution has again been urged in the description of any photon by adjectival categorization; in particular the intricacy of relationship between spin and multipolar order can be misrepresented by simplistic terminology. In much the same way, loose usage of the term 'photon wavefunction' is suggestive of a mathematical representation that is quite remote from what robust theory will allow. It is hoped that in the continuing discussion of such concerns, the community can better recognize the limitations of some current terminology, and so advance a truer comprehension of the reality of the photon.

\section{REFERENCES}

[1] D. S. Roychoudhuri, A. F. Kracklauer, and K. Creath, [The Nature of Light. What is a Photon?] CRC Press, Boca Raton, (2008).

[2] P. W. Milonni, [The Quantum Vacuum: An Introduction to Quantum Electrodynamics] Academic Press, Boston, Mass. ; London, (1994).

[3] G. Gabrielse, D. Hanneke, T. Kinoshita, M. Nio, and B. Odom, "New determination of the fine structure constant from the electron $g$ value and QED," Physical Review Letters 97, 030802 (2006).

[4] D. P. Craig and T. Thirunamachandran, [Molecular Quantum Electrodynamics. An Introduction to RadiationMolecule Interactions] Dover, Mineola; New York, (1998).

[5] E. A. Power, and T. Thirunamachandran, "Quantum electrodynamics with nonrelativistic sources. IV. Poynting vector, energy densities, and other quadratic operators of the electromagnetic field," Physical Review A 45, 54-63 (1992).

[6] M. A. Lopez-Marino, and J. L. Jimenez, "Analysis of the Abraham-Minkowski controversy by means of two simple examples," Foundations of Physics Letters 17, 1-23 (2004).

[7] M. Mansuripur, "Radiation pressure and the linear momentum of light in dispersive dielectric media," Optics Express 13, 2245-2250 (2005).

[8] R. Loudon, S. M. Barnett and C. Baxter, "Radiation pressure and momentum transfer in dielectrics: The photon drag effect," Physical Review A 71, 063802 (2005).

[9] R. N. C. Pfeifer, T. A. Nieminen, N. R. Heckenberg, and H. Rubinsztein-Dunlop, "Two controversies in classical electromagnetism," Proceedings of the SPIE 6326, 63260H (2006).

[10] M. Scalora, G. D'Aguanno, N. Mattiucci, M. J. Bloemer, M. Centini, C. Sibilia, and J. W. Haus, "Radiation pressure of light pulses and conservation of linear momentum in dispersive media," Physical Review E 73, 056604 (2006).

[11] L. Mandel, and E. Wolf, [Optical Coherence and Quantum Optics] University Press, Cambridge, (1995). 
[12] C. Cohen-Tannoudji, J. Dupont-Roc, and G. Grynberg, [Photons and Atoms. Introduction to Quantum Electrodynamics] John Wiley, New York (1989).

[13] S. J. Enk, and G Nienhuis, "Spin and angular momentum of photons," Europhysics Letters 25, 497-501 (1994).

[14] D. L. Andrews, [Structured Light and Its Applications. An Introduction to Phase-Structured Beams and Nanoscale Optical Forces] Academic Press, Amsterdam (2008).

[15] J. Leach, E. Yao, and M. J. Padgett, “Observation of the vortex structure of a non-integer vortex beam," New Journal of Physics 6, 71 (2004).

[16] J. B. Gotte, S. Franke-Arnold, and R. Zambrini, "Quantum formulation of fractional orbital angular momentum,” Journal of Modern Optics 54, 1723-1738 (2007).

[17] M. Mansuripur, "Angular momentum of circularly polarized light in dielectric media," Optics Express 13, 5315-5324 (2005).

[18] M. Ohtsu, K. Kobayashi, T. Kawazoe, T. Yatsui, and M. Naruse, [Principles of Nanophotonics] CRC Press, Boca Raton (2008).

[19] A. Muthukrishnan, M. O. Scully, and M. S. Zubairy, "The photon wave function," Proceedings of the SPIE 5866, 287-292 (2005).

[20] M. G. Rayner, and B. J. Smith,'The Maxwell wave function of the photon," Proceedings of the SPIE 5866, 293297 (2005).

[21] M. Ligare, and R. Oliveri, "The calculated photon: Visualization of a quantum field," American Journal of Physics 70, 58-66 (2002).

[22] P. Grangier, J. A. Levenson, and J.-P. Poizat, "Quantum non-demolition measurements in optics," Nature 396, 537-542 (1998).

[23] S. Gleyzes, S. Kuhr, C. Guerlin, J. Bernu, S. Deleglise, U. B. Hoff, M. Brune, J. M. Raimond, and S. Haroche, "Quantum jumps of light recording the birth and death of a photon in a cavity," Nature 446, 297-300 (2007).

[24] F. Halzen, and A.D. Martin, [Quarks and Leptons] Wiley, New York (1984).

[25] R. Loudon, [The Quantum Theory of Light] 3rd edn. University Press, Oxford (2000).

[26] M. Salem, and E. Wolf, "Coherence-induced polarization changes in light beams," Optics Letters 33, 1180$1182(2008)$.

[27] D. L. Andrews, "The photon: a virtual reality," Proceedings of the SPIE 5866, 1-7 (2005).

[28] V. B. Berestetskii, E. M. Lifshitz, and L. P. Pitaevskii, [Quantum Electrodynamics] 2nd edn. Butterworth, London (1982).

[29] D. L. Andrews, work in progress.

[30] A. A. Demidov, private communication.

[31] G. D. Scholes and D. L. Andrews, "Damping and higher multipole effects in the quantum electrodynamical model for electronic energy transfer in the condensed phase," Journal of Chemical Physics 107, 5374-5384 (1997).

[32] A. Salam, "A general formula for the rate of resonant transfer of energy between two electric multipole moments of arbitrary order using molecular quantum electrodynamics," Journal of Chemical Physics 122, 044112 (2005). 